\title{
The number of unsieved integers up to $x$
}

\author{
by \\ ANDREW Granville (Montréal) \\ and K. Soundararajan (Ann Arbor, MI)
}

1. Introduction. Let $f$ denote a multiplicative function with $0 \leq$ $f(n) \leq 1$ for all positive integers $n$. Define

$$
\Theta(f, x):=\prod_{p \leq x}\left(1-\frac{1}{p}\right)\left(1+\frac{f(p)}{p}+\frac{f\left(p^{2}\right)}{p^{2}}+\cdots\right) .
$$

Given a real number $w \geq 1$ in this paper we are concerned with the problem of determining

$$
g(w):=\liminf _{x \rightarrow \infty} \frac{1}{x} \sum_{n \leq x} f(n) \quad \text { and } \quad G(w):=\limsup _{x \rightarrow \infty} \frac{1}{x} \sum_{n \leq x} f(n),
$$

where both limits are taken over the class of multiplicative functions $f$ with $\Theta(f, x)=1 / w+o(1)$. (To be more precise, suppose that $\varepsilon(x)$ is a positive-valued function for which $\varepsilon(x) \rightarrow 0$ as $x \rightarrow \infty$. Define $g(w, x):=$ $\min (1 / x) \sum_{n \leq x} f(n)$ and $G(w, x):=\max (1 / x) \sum_{n \leq x} f(n)$, where the "min" and "max" are taken over all multiplicative $f$, with $0 \leq f(n) \leq 1$ for all $n \geq 1$, and $1-\varepsilon(x)<w \Theta(f, x)<1+\varepsilon(x)$. Then let $g(w):=\liminf _{x \rightarrow \infty} g(w, x)$ and $G(w):=\limsup _{x \rightarrow \infty} G(w, x)$.)

P. Erdős and I. Ruzsa [1] showed that $g(w)>0$ for all $w$. Consider the function $f$ with $f\left(p^{k}\right)=1$ for $p \leq x^{1 / w}$ and $f\left(p^{k}\right)=0$ for $x^{1 / w} \leq p \leq x$. Then one has $\Theta(f ; x)=1 / w+o(1)$ and further $\sum_{n \leq x} f(n)=\psi\left(x, x^{1 / w}\right)$, the number of integers below $x$ having no prime factors above $x^{1 / w}$. It is well known that for any fixed $w$ we have

$$
\psi\left(x, x^{1 / w}\right)=x \varrho(w)\left(1+O\left(\frac{w}{\log x}\right)\right),
$$

2000 Mathematics Subject Classification: Primary 11N60; Secondary 11N35, 11N37.

Le premier auteur est partiellement soutenu par une bourse du Conseil de recherches en sciences naturelles et en génie du Canada. The second author is partially supported by the National Science Foundation and the American Institute of Mathematics (AIM). 
where $\varrho(w)$ is the Dickman-de Bruijn function, defined by $\varrho(w)=1$ for $0 \leq w \leq 1$, and $w \varrho^{\prime}(w)=-\varrho(w-1)$ for all $w \geq 1$. This example shows that $g(w) \leq \varrho(w)$ and A. Hildebrand [6] established that in fact $g(w)=\varrho(w)$. Since $\varrho(w)=w^{-w+o(w)}$ note that $g(w)$ decays very rapidly as $w$ increases.

Regarding $G(w)$, R. Hall [4] established that $G(w) \leq e^{\gamma} / w$ and Hildebrand [5] improved this slightly by showing that $G(w) \leq w^{-1} \int_{0}^{w} \varrho(t) d t$. Since $\int_{0}^{\infty} \varrho(t) d t=e^{\gamma}$ this does mark an improvement over Hall's result, but the difference from $e^{\gamma} / w$ is $w^{-1} \int_{w}^{\infty} \varrho(t) d t=w^{-w+o(w)}$, which is very small. In this paper we shall prove that $G(w)=e^{\gamma} / w-1 / w^{2+o(1)}$, but it remains to determine $G(w)$ more precisely. We shall also give a shorter proof of Hildebrand's result that $g(w)=\varrho(w)$.

TheOREM 1. For all $w \geq 1$ we have

$$
G(w) \geq \max _{w \geq \Delta \geq 0}\left(\varrho(w+\Delta)+\int_{0}^{\Delta} \frac{\varrho(t)}{w+\Delta-t} d t\right) .
$$

When $w$ is large, the maximum is attained for $\Delta \sim \log w / \log \log w$, and yields

$$
G(w) \geq \frac{e^{\gamma}}{w}-\frac{\left(e^{\gamma}+o(1)\right) \log w}{w^{2} \log \log w} .
$$

TheORem 2. For all large $w$ we have

$$
G(w) \leq \frac{e^{\gamma}}{w}-\frac{1}{w^{2} \exp \left(c(\log w)^{2 / 3}(\log \log w)^{1 / 3}\right)}
$$

for a positive constant $c$.

We also give an explicit upper bound for $G(w)$ valid for all $w$.

Theorem 3. For $1 \leq w$ we have

$$
G(w) \leq 1-\log w+(\log w)^{2} / 2
$$

and equality holds here for $1 \leq w \leq 3 / 2$. For $w \geq 1$ put

$$
\Lambda(w):=\frac{1}{2}(w+1 / w)+\frac{\log w}{2}(w-1 / w) .
$$

Then

$$
G(w) \leq \Lambda(w) \log \left(1+e^{\gamma} /(w \Lambda(w))\right) .
$$

The first bound in Theorem 3 is better than the second for $w \leq 3.21 \ldots$, when the second bound takes over. Note that the second bound in Theorem 3 equals $e^{\gamma} / w-\left(e^{2 \gamma}+o(1)\right) / w^{3} \log w$, only a little weaker than the bound in Theorem 2, while being totally explicit.

In the range $1 \leq w \leq 3 / 2$ we may check that the right side of (1.3) equals $1-\log w+(\log w)^{2} / 2=G(w)$. Perhaps it is true that $G(w)$ is given by the right side of (1.3) for all $w$. 
We end this section by giving a simple construction that proves Theorem 1.

Proof of Theorem 1. Let $y$ be large and consider the completely multiplicative function $f$ defined by $f(p)=0$ for $p \in\left[y, y^{w}\right]$ and $f(p)=1$ for all other primes $p$. Put $x=y^{w+\Delta}$ where $0 \leq \Delta \leq w$ and note that $\Theta(f, x)=\prod_{y \leq p \leq y^{w}}(1-1 / p) \sim 1 / w$. An integer $n \leq x$ with $f(n)=1$ has at most one prime factor between $y^{w}$ and $x$, and all its other prime factors are below $y$. Hence

$$
\sum_{n \leq x} f(n)=\psi(x, y)+\sum_{y^{w} \leq p \leq x} \psi(x / p, y),
$$

and by (1.2) and the prime number theorem this is

$$
\begin{aligned}
& \sim x \varrho(w+\Delta)+x \sum_{y^{w} \leq p \leq x} \frac{1}{p} \varrho\left(w+\Delta-\frac{\log p}{\log y}\right) \\
& \sim x\left(\varrho(w+\Delta)+\int_{0}^{\Delta} \frac{\varrho(t)}{w+\Delta-t} d t\right),
\end{aligned}
$$

which gives the lower bound (1.3) for $G(w)$. For large $w$ we see that

$$
\begin{aligned}
\varrho(w+\Delta) & +\int_{0}^{\Delta} \frac{\varrho(t)}{w+\Delta-t} d t \\
& =\frac{1}{w+\Delta} \int_{0}^{\Delta} \varrho(t) d t+\int_{0}^{\Delta} \frac{t \varrho(t)}{(w+\Delta)(w+\Delta-t)} d t+\varrho(w+\Delta)
\end{aligned}
$$

and since $\int_{0}^{\infty} t \varrho(t) d t<\infty$ and $\int_{0}^{\Delta} \varrho(t) d t=e^{\gamma}-\Delta^{-(1+o(1)) \Delta}$ the above is

$$
\frac{1}{w+\Delta}\left(e^{\gamma}-\Delta^{-(1+o(1)) \Delta}\right)+O\left(\frac{1}{w^{2}}\right) \text {. }
$$

The quantity above attains a maximum for $\Delta=(1+o(1)) \log w / \log \log w$, completing the proof of Theorem 1.

We noted above that $G(w)=1-\log w+(\log w)^{2} / 2$ for $1 \leq w \leq 1.5$ (with the maximum attained in (1.3) at $\Delta=w$ ). Next we record the bounds obtained for $1.5 \leq w \leq 2$ (though here the maximum is attained with $\Delta$ a little smaller than $w$ ).

The upper and lower bounds for $G(w)$ given by Theorems 1 and 3

\begin{tabular}{c|cccccc}
\hline$w$ & 1.5 & 1.6 & 1.7 & 1.8 & 1.9 & 2.0 \\
\hline$G(w) \geq$ & .676735 & .640255 & .608806 & .581685 & .557392 & .535905 \\
$G(w) \leq$ & .676736 & .640449 & .610155 & .584960 & .564135 & .547080 \\
\hline
\end{tabular}


2. Reformulation in terms of integral equations. E. Wirsing [8] observed that questions on mean values of multiplicative functions can be reformulated in terms of solutions to a certain integral equation. We formalized this connection precisely in our paper [2] and we now recapitulate the salient details. We will prove our results by establishing the corresponding statements for solutions to integral equations.

The following class of integral equations is relevant to the study of multiplicative functions $f$ with $|f(n)| \leq 1$ for all $n$ : Let $\chi$ be a measurable function with $\chi(t)=1$ for $t \leq 1$ and $|\chi(t)| \leq 1$ for all $t \geq 1$. Let $\sigma(u)=1$ for $u \leq 1$ and for $u>1$ we define $\sigma$ to be the solution to

$$
u \sigma(u)=\int_{0}^{u} \chi(t) \sigma(u-t) d t .
$$

In [2] we showed that there is a unique continuous solution $\sigma(u)$ to $(2.1)$ and that $|\sigma(u)| \leq 1$ for all $u$. In fact $\sigma(u)$ is given by

$$
\sigma(u)=1+\sum_{j=1}^{\infty} \frac{(-1)^{j}}{j !} I_{j}(u ; \chi)
$$

where

$$
I_{j}(u ; \chi)=\int_{\substack{t_{1}, \ldots, t_{j} \geq 1 \\ t_{1}+\cdots+t_{j} \leq u}} \frac{1-\chi\left(t_{1}\right)}{t_{1}} \cdots \frac{1-\chi\left(t_{j}\right)}{t_{j}} d t_{1} \cdots d t_{j} .
$$

The connection between multiplicative functions and the integral equation (2.1) is given by the following result, which is Proposition 1 in [2].

Proposition 2.1. Let $f$ be a multiplicative function with $|f(n)| \leq 1$ for all $n$, and $f(n)=1$ for $n \leq y$. Let $\vartheta(x)=\sum_{p \leq x} \log p$ and define

$$
\chi(u)=\chi_{f}(u)=\frac{1}{\vartheta\left(y^{u}\right)} \sum_{p \leq y^{u}} f(p) \log p .
$$

Then $\chi(t)$ is a measurable function with $|\chi(t)| \leq 1$ for all $t$ and $\chi(t)=1$ for $t \leq 1$. Let $\sigma(u)$ be the corresponding unique solution to (2.1). Then

$$
\frac{1}{y^{u}} \sum_{n \leq y^{u}} f(n)=\sigma(u)+O\left(\frac{u}{\log y}+\frac{1}{y^{u}}\right) .
$$

For our problems on non-negative multiplicative functions we will restrict attention to integral equations where $\chi(t)$ only takes values in $[0,1]$. The corresponding solution $\sigma(u)$ to $(2.1)$ then also takes values in $[0,1]$. We also define

$$
E(u)=E_{\chi}(u):=\exp \left(\int_{0}^{u} \frac{1-\chi(t)}{t} d t\right) .
$$


Notice that $\Theta\left(f, y^{u}\right) \sim 1 / E(u)$ when $\chi$ is defined as in Proposition 2.1. Analogously to $g(w)$ and $G(w)$ we may define

$$
\widetilde{g}(w)=\liminf _{\substack{u, \chi \\ E_{\chi}(u)=w}} \sigma(u) \text { and } \widetilde{G}(w)=\limsup _{\substack{u, \chi \\ E_{\chi}(u)=w}} \sigma(u),
$$

where the limits are taken over all pairs $u, \chi$ with $u \geq 1$, where $\chi$ is a measurable function for which $\chi(t)=1$ for $t \leq 1$ and $\chi(t) \in[0,1]$ for all $t$, and with $E_{\chi}(u)=w$. We shall show that these quantities are in fact equal to $g(w)$ and $G(w)$ respectively. Something similar was stated (but not very precisely) by Hildebrand in his discussion paper [7].

Theorem 2.2. We have $g(w)=\widetilde{g}(w)$ and $G(w)=\widetilde{G}(w)$.

To prove Theorem 2.2 we need to know how small primes affect the mean values of multiplicative functions, so that we can remove their effect and be in a position to use Proposition 2.1. We also require a converse to Proposition 2.1 which allows us to go from integral equations to multiplicative functions. Such results were established in [2] and we now quote them in our context. Proposition 4.4 of [2] (with $\varphi=\pi / 2$ there) gives the following lemma.

Lemma 2.3. Let $f$ be a multiplicative function with $0 \leq f(n) \leq 1$ for all $n$. Let $1 \geq \varepsilon \geq \log 2 / \log x$ and take $g$ to be the completely multiplicative function with $g(p)=1$ if $p \leq x^{\varepsilon}$ and $g(p)=f(p)$ otherwise. Then

$$
\frac{1}{x} \sum_{n \leq x} f(n)=\Theta\left(f, x^{\varepsilon}\right) \frac{1}{x} \sum_{m \leq x} g(m)+O\left(\varepsilon^{1 / 4-1 / 2 \pi}\right) .
$$

Next, Proposition 1 (Converse) in [2] gives the following converse to Proposition 2.1.

Proposition 2.4. Let $\chi$ be a given measurable function with $\chi(t)=1$ for $t \leq 1$ and $\chi(t) \in[0,1]$ for all $t \geq 1$ and let $\sigma$ denote the corresponding solution to (2.1). Given $\varepsilon>0$ and $u \geq 1$ there exist arbitrarily large $y$ and a multiplicative function $f$ with $f(n)=1$ for $n \leq y$ and $0 \leq f(n) \leq 1$ for all $n$ and with

$$
\left|\chi(t)-\frac{1}{\vartheta\left(y^{t}\right)} \sum_{p \leq y^{t}} f(p) \log p\right| \leq \varepsilon \quad \text { for almost all } 0 \leq t \leq u .
$$

Further, for all $1 \leq t \leq u$,

$$
\sigma(t)=\frac{1}{y^{t}} \sum_{n \leq y^{t}} f(n)+O\left(u^{\varepsilon}-1\right)+O\left(\frac{u}{\log y}\right) .
$$

We will defer the proof of Theorem 2.2 to the next section. But let us note that combining Lemma 2.3 with Proposition 2.1 gives 


$$
g(w) \geq \min _{w \geq v \geq 1} \frac{1}{v} \widetilde{g}\left(\frac{w}{v}\right) \text { and } G(w) \leq \max _{w \geq v \geq 1} \frac{1}{v} \widetilde{G}\left(\frac{w}{v}\right) .
$$

Also from Proposition 2.4 we get

$$
g(w) \leq \widetilde{g}(w), \quad \widetilde{G}(w) \leq G(w) .
$$

We end this section by recording two facts which will be useful in our later work. Firstly when $\chi(t) \in[0,1]$ one obtains inclusion-exclusion inequalities from $(2.2 \mathrm{a}, \mathrm{b})$ : namely, for all even integers $n$ we have (see Proposition 3.6 of $[2])$

$$
\sum_{j=0}^{n} \frac{(-1)^{j}}{j !} I_{j}(u ; \chi) \geq \sigma(u) \geq \sum_{j=0}^{n+1} \frac{(-1)^{j}}{j !} I_{j}(u ; \chi) .
$$

Secondly from $(2.2 \mathrm{a}, \mathrm{b})$ and a little combinatorics we obtain the following lemma (see Lemma 3.4 of [2]):

Lemma 2.5. Let $\chi$ and $\widehat{\chi}$ be two measurable functions with $\chi(t)=\widehat{\chi}(t)=1$ for $0 \leq t \leq 1$ and $|\chi(t)|,|\widehat{\chi}(t)| \leq 1$ for all $t$. Let $\sigma$ and $\widehat{\sigma}$ be the corresponding solutions to (2.1). Then $\widehat{\sigma}(u)$ equals

$$
\begin{aligned}
\sigma(u)+\sum_{j=1}^{\infty} \frac{1}{j !} \int_{\substack{t_{1}, \ldots, t_{j} \geq 1 \\
t_{1}+\cdots+t_{j} \leq u}} \frac{\widehat{\chi}\left(t_{1}\right)-\chi\left(t_{1}\right)}{t_{1}} & \cdots \frac{\widehat{\chi}\left(t_{j}\right)-\chi\left(t_{j}\right)}{t_{j}} \\
& \times \sigma\left(u-t_{1}-\cdots-t_{j}\right) d t_{1} \cdots d t_{j} .
\end{aligned}
$$

3. Upper bounds for $G(w)$ and Lipschitz estimates. For a measurable function $g:[0, \infty) \rightarrow \mathbb{C}$ we will denote the Laplace transform of $g$ by $\mathcal{L}(g, s):=\int_{0}^{\infty} g(t) e^{-s t} d t$. If $g$ is integrable and grows sub-exponentially (that is, for every $\varepsilon>0,|g(t)| \ll_{\varepsilon} e^{\varepsilon t}$ almost everywhere) then the Laplace transform is well defined for all complex numbers $s$ with $\operatorname{Re}(s)>0$. Integrating term by term in $(2.2 \mathrm{a}, \mathrm{b})$ we see that

$$
\mathcal{L}(\sigma, s)=\frac{1}{s} \exp \left(-\mathcal{L}\left(\frac{1-\chi(v)}{v}, s\right)\right) .
$$

Suppose now that $\chi(t)=1$ for $t \leq 1$ and $\chi(t) \in[0,1]$ for all $t$ and we are given $u \geq 1$. Define $\widehat{\chi}(t)=\chi(t)$ for $t \leq u$ and $\widehat{\chi}(t)=0$ for $t>u$. If $\sigma$ and $\widehat{\sigma}$ are the corresponding solutions to (2.1) then note that $\sigma(v)=\widehat{\sigma}(v)$ for $v \leq u$ and that $E_{\chi}(v)=E_{\widehat{\chi}}(v)$ for $v \leq u$. Now

$$
\begin{aligned}
\sigma(u)=\widehat{\sigma}(u) & =\frac{1}{u} \int_{0}^{u} \widehat{\sigma}(t) \widehat{\chi}(u-t) d t \\
& \leq \frac{1}{u} \int_{0}^{u} \widehat{\sigma}(t) d t=\frac{1}{u} \int_{0}^{\infty} \widehat{\sigma}(t) d t-\frac{1}{u} \int_{u}^{\infty} \widehat{\sigma}(t) d t .
\end{aligned}
$$


Further

$$
\begin{aligned}
\mathcal{L}\left(\frac{1-\widehat{\chi}(t)}{t}, s\right)-\log E(u) & =\int_{0}^{\infty}\left(\frac{1-\widehat{\chi}(t)}{t}\right) e^{-s t} d t-\int_{0}^{u} \frac{1-\chi(t)}{t} d t \\
& =\int_{0}^{u}\left(\frac{1-\chi(t)}{t}\right)\left(e^{-s t}-1\right) d t+\int_{u}^{\infty} \frac{e^{-s t}}{t} d t \\
& =-\gamma-\log (s u)+O(u|s|)
\end{aligned}
$$

for small $s$, since $\gamma=\int_{0}^{1} \frac{1-e^{-t}}{t} d t-\int_{1}^{\infty} \frac{e^{-t}}{t} d t$. Hence

$$
\frac{1}{u} \int_{0}^{\infty} \widehat{\sigma}(t) d t=\frac{1}{u} \lim _{y \rightarrow 0} \mathcal{L}(\widehat{\sigma}, y)=\lim _{y \rightarrow 0} \frac{1}{y u} \exp \left(-\mathcal{L}\left(\frac{1-\widehat{\chi}(t)}{t}, y\right)\right)=\frac{e^{\gamma}}{E(u)},
$$

and so we have

$$
\sigma(u) \leq \frac{e^{\gamma}}{E(u)}-\frac{1}{u} \int_{u}^{\infty} \widehat{\sigma}(t) d t .
$$

We use (3.2) in the proofs of Theorems 2 and 3, since it allows us to give an upper bound for $\sigma(u)$ by determining a "smoothed lower bound" for $\widehat{\sigma}$. Our plan for proving a bound on this integral is to bound how much $\widehat{\sigma}(t)$ changes as $t$ gets bigger than $u$, via Lipschitz-type estimates.

For general complex $\chi$ with $|\chi| \leq 1$, and $\sigma$ satisfying (2.1), we might expect to have a Lipschitz estimate of the form

$$
|| \sigma(u)|-| \sigma(v)|| \ll\left(\frac{u-v}{u}\right)^{\kappa}\left(1+\log \frac{u}{u-v}\right) \text { whenever } 1 \leq v \leq u
$$

for certain values of $\kappa>0$; and indeed we established (3.3) in [3] for $\kappa=$ $1-2 / \pi$. Any increase in the value of $\kappa$ allows stronger consequences, and we believe that $\kappa=1$ in (3.3) is probably valid. Note that no exponent $>1$ is possible since $|\varrho(1+\delta)-\varrho(1)|=\log (1+\delta) \sim \delta$ for $0 \leq \delta \leq 1$. We are able to improve " $1-2 / \pi$ " to " $1-1 / \pi$ " in the special case that $\chi(t) \in[0,1]$ for all $t$.

THEOREM 4. Let $\chi$ be a measurable function with $\chi(t)=1$ for $t \leq 1$ and $\chi(t) \in[0,1]$ for $t>1$, and let $\sigma$ denote the corresponding solution to (2.1). Then

$$
|\sigma(u)-\sigma(v)| \ll\left(\frac{u-v}{u}\right)^{1-1 / \pi}\left(1+\log \frac{u}{u-v}\right) \quad \text { whenever } 1 \leq v \leq u .
$$

Theorem 4 follows immediately from the stronger but more complicated Proposition 4.2 below, and the fact that $|\sigma(u)-\sigma(v)| \leq 3(u-v) / u$ whenever $v \leq u(1-1 / E(u))$. This is trivial for $v \leq 2 u / 3$, whereas for larger $v$ in the 
range, we obtain

$$
|\sigma(u)-\sigma(v)| \leq \frac{e^{\gamma}}{E(v)} \leq \frac{u e^{\gamma}}{v E(u)} \leq \frac{3(u-v)}{u},
$$

using Hall's result that $\sigma(u) \leq e^{\gamma} / E(u)$.

Using (3.3) in (3.2) leads to the bound

$$
\widetilde{G}(w) \leq e^{\gamma} / w-C_{\kappa} /\left(w^{1+1 / \kappa} \log w\right)
$$

for some positive constant $C_{\kappa}$. Thus if (3.3) holds with $\kappa=1$ then we would be able to deduce that $G(w)=e^{\gamma} / w-(\log w)^{O(1)} / w^{2}$ by Theorem 1 .

In order to prove Theorem 3 we give the following explicit Lipschitz estimate (see also Proposition 4.1 of [2]).

Proposition 3.1. Let $\chi$ be a measurable function with $\chi(t)=1$ for $t \leq 1$ and $\chi(t) \in[0,1]$ for all $t$, and let $\sigma(u)$ denote the corresponding solution to (2.1). Then for all $u \geq 1$ and $1 \geq \delta>0$ we have

$\log (1+\delta)\left(\frac{E(u)-1 / E(u)}{2}+\log E(u) \frac{E(u)+1 / E(u)}{2}\right) \geq \sigma(u(1+\delta))-\sigma(u)$ and

$$
\begin{aligned}
& \sigma(u(1+\delta))-\sigma(u) \\
& \quad \geq-\log (1+\delta)\left(\frac{E(u)+1 / E(u)}{2}+\log E(u) \frac{E(u)-1 / E(u)}{2}\right) .
\end{aligned}
$$

Proof. We shall only prove the lower bound, the proof of the upper bound is similar. From $(2.2 \mathrm{a}, \mathrm{b})$ we see that

$$
\sigma(u(1+\delta))-\sigma(u) \geq-\sum_{\substack{j=1 \\ j \text { odd }}}^{\infty} \frac{1}{j !}\left(I_{j}(u(1+\delta) ; \chi)-I_{j}(u ; \chi)\right) .
$$

By symmetry we see that $I_{j}(u(1+\delta) ; \chi)-I_{j}(u ; \chi)$ equals

$$
\begin{gathered}
j \int_{t_{1}, \ldots, t_{j-1} \geq 1} \frac{1-\chi\left(t_{1}\right)}{t_{1}} \cdots \frac{1-\chi\left(t_{j-1}\right)}{t_{j-1}} \\
\times \int_{\substack{\max \left(t_{1}, \ldots, t_{j-1}, u-t_{1}-\cdots-t_{j-1}\right) \leq t_{j} \\
t_{j} \leq u(1+\delta)-t_{1}-\cdots-t_{j-1}}} \frac{1-\chi\left(t_{j}\right)}{t_{j}} d t_{1} \cdots d t_{j} .
\end{gathered}
$$

The integral over $t_{j}$ is

$$
\leq \log \frac{u / j+u \delta}{u / j}=\log (1+j \delta) \leq j \log (1+\delta)
$$

since $\max \left(t_{1}, \ldots, t_{j-1}, u-t_{1}-\cdots-t_{j-1}\right) \geq u / j$. Further since $\delta<1$ we have $t_{1}, \ldots, t_{j-1} \leq u$ and so these integrals contribute $\leq(\log E(u))^{j-1}$. Thus we 
have

$$
\sigma(u(1+\delta))-\sigma(u) \geq-\sum_{\substack{j=1 \\ j \text { odd }}}^{\infty} \frac{1}{j !} j^{2} \log (1+\delta)(\log E(u))^{j-1},
$$

and the result follows easily.

Proof of Theorem 2.2. Fix $w \geq v \geq 1$. Suppose $\chi(t)=1$ for $t \leq 1$ and $\chi(t) \in[0,1]$ for all $t$ and let $\sigma(u)$ denote the corresponding solution to (2.1) (we will think of $\chi$ as giving the optimal function for either $\widetilde{g}(w / v)$ or $\widetilde{G}(w / v))$. Let $U \geq 1$ be a parameter which we will let tend to infinity. Put $\chi_{1}(t)=\chi(t / U)$ and note that the corresponding solution to (2.1) is $\sigma_{1}(u)=\sigma(u / U)$. Define $\chi_{2}(t)=0$ for $1 \leq t \leq v$ and $\chi_{2}(t)=\chi_{1}(t)$ for all other $t$, and let $\sigma_{2}(u)$ denote the corresponding solution to (2.1). By Lemma 2.5 we see that for $U \geq v$,

$$
\begin{aligned}
\sigma_{2}(u U)= & \sigma_{1}(u U)+\sum_{j=1}^{\infty} \frac{(-1)^{j}}{j !} \int_{\substack{v \geq t_{1}, \ldots, t_{j} \geq 1 \\
t_{1}+\cdots+t_{j} \leq u U}} \frac{1}{t_{1}} \cdots \frac{1}{t_{j}} \\
& \times \sigma_{1}\left(u U-t_{1}-\cdots-t_{j}\right) d t_{1} \cdots d t_{j} .
\end{aligned}
$$

By Proposition 3.1 we know that

$$
\sigma_{1}\left(u U-t_{1}-\cdots-t_{j}\right)=\sigma_{1}(u U)+O\left(\min \left(1, E_{\chi}(u) \log E_{\chi}(u) \frac{j v}{u U}\right)\right) .
$$

Using this above we easily see that for large $U$ with $u, v, w$ fixed we have $\sigma_{2}(u U) \sim \sigma_{1}(u U) / v=\sigma(u) / v$ and note further that $E_{\chi_{2}}(u U)=v E_{\chi_{1}}(u U)$ $=v E_{\chi}(u)$.

This scaling argument shows that for $1 \leq v \leq w$ we have $\widetilde{g}(w / v) \geq v \widetilde{g}(w)$ and that $\widetilde{G}(w / v) \leq v \widetilde{G}(w)$. Using these inequalities in (2.4a) we deduce that $g(w) \geq \widetilde{g}(w)$ and that $G(w) \leq \widetilde{G}(w)$ and combining this with $(2.4 \mathrm{~b})$ we obtain Theorem 2.2.

Now that Theorem 2.2 has been established, to prove Theorem 3 it suffices to establish the analogous bounds for $\widetilde{G}(w)$ and we establish these next.

Proof of Theorem 3. Using the inclusion-exclusion upper bound (2.5) with $n=2$ we see that $\sigma(u) \leq 1-\log E(u)+(\log E(u))^{2} / 2$. It follows that $G(w)=\widetilde{G}(w) \leq 1-\log w+(\log w)^{2} / 2$. If $w \leq 3 / 2$ then consider $\chi(t)=0$ for $1 \leq t \leq w$ and $\chi(t)=1$ for all other $t$. Then we see that the corresponding solution $\sigma(u)$ satisfies $\sigma(u)=1-\log w+(\log w)^{2} / 2$ for $3 \geq u \geq 2 w$. Thus $\widetilde{G}(w)=1-\log w+(\log w)^{2} / 2$ for $1 \leq w \leq 3 / 2$.

We now establish the second bound of the theorem. As noted in the introduction the second bound is worse than the first for $w \leq 3.21$ and so 
we may suppose that $w \geq 2$. With $\widehat{\chi}, \widehat{\sigma}$ as above, note that $\widehat{\sigma}(t) \geq 0$ for all $t$, and

$$
\widehat{\sigma}(u(1+\delta)) \geq \widehat{\sigma}(u)-\Lambda(E(u)) \log (1+\delta) \quad \text { for } 0 \leq \delta \leq 1
$$

by Proposition 3.1. If $E(u) \geq 2$ then $\Lambda(E(u)) \geq 7 / 4>1 / \log 2$ so that $\exp (\sigma(u) / \Lambda(E(u)))-1<1$. Hence we obtain

$$
\begin{aligned}
\frac{1}{u} \int_{u}^{\infty} \widehat{\sigma}(t) d t & \geq \int_{0}^{\exp (\sigma(u) / \Lambda(E(u)))-1}(\sigma(u)-\Lambda(E(u)) \log (1+\delta)) d \delta \\
& =-\sigma(u)+\Lambda(E(u))\left(\exp \left(\frac{\sigma(u)}{\Lambda(E(u))}\right)-1\right),
\end{aligned}
$$

and inserting this into (3.2) we get the theorem.

4. An improved upper bound: Proof of Theorem 2. Our proof of Theorem 2 is also based on (3.2) and on obtaining lower bounds for $u^{-1} \int_{u}^{\infty} \widehat{\sigma}(t) d t$. However Theorem 4 is not quite strong enough to obtain this conclusion and so, in this section, we develop a hybrid Lipschitz estimate which for our problem is almost as good as (3.3) with $\kappa=1$. We begin with the following proposition (compare Lemma 2.2 and Proposition 3.3 of [3]).

Proposition 4.1. Let $\chi$ be a measurable function with $\chi(t)=1$ for $t \leq 1$ and $\chi(t)$ in the unit disc for all $t$. Let $\sigma$ be the corresponding solution to (2.1). Let $1 \leq v \leq u$ be given real numbers, and put $\delta=u-v$. Define

$$
F:=\max _{y \in \mathbb{R}} \exp \left(\gamma-\int_{0}^{u} \operatorname{Re}\left(\frac{1-\chi(t) e^{-i t y}}{t}\right) d t\right)\left|1-e^{-i y \delta}\right| .
$$

Then

$$
\begin{aligned}
|\sigma(u)-\sigma(v)| & \leq \frac{\delta}{u} \log \frac{e u}{\delta}+F+F \int_{0}^{2 /(u F)} \frac{1-e^{-2 x u}}{x} d x \\
& \leq \frac{\delta}{u} \log \frac{e u}{\delta}+F \log \frac{e^{3}}{F}
\end{aligned}
$$

Proof. As in the proof of Theorem 3 take $\widehat{\chi}(t)=\chi(t)$ for $t \leq u$ and $\widehat{\chi}(t)=0$ for $t>u$, and let $\widehat{\sigma}$ be the corresponding solution to (2.1). Set $\sigma(t)=\widehat{\sigma}(t)=0$ for $t<0$. Note that

$$
\begin{aligned}
|u \sigma(u)-v \sigma(v)| & =|u \widehat{\sigma}(u)-v \widehat{\sigma}(v)|=\left|\int_{0}^{u} \chi(t)(\widehat{\sigma}(u-t)-\widehat{\sigma}(v-t)) d t\right| \\
\leq & \int_{0}^{u}|\widehat{\sigma}(t)-\widehat{\sigma}(t-\delta)| d t=\int_{0}^{u} 2 t|\widehat{\sigma}(t)-\widehat{\sigma}(t-\delta)|\left(\int_{0}^{\infty} e^{-2 x t} d x\right) d t
\end{aligned}
$$




$$
\begin{aligned}
& \leq 2 \int_{0}^{\infty} \int_{0}^{u}\{|t \widehat{\sigma}(t)-(t-\delta) \widehat{\sigma}(t-\delta)|+\delta|\widehat{\sigma}(t-\delta)|\} e^{-2 t x} d t d x \\
& \leq \int_{0}^{\infty} I(x) d x+\int_{0}^{\infty} \int_{\delta}^{u} 2 \delta e^{-2 t x} d t d x=\delta \log \frac{u}{\delta}+\int_{0}^{\infty} I(x) d x
\end{aligned}
$$

where

$$
I(x)=\int_{0}^{u} 2|t \widehat{\sigma}(t)-(t-\delta) \widehat{\sigma}(t-\delta)| e^{-2 t x} d t
$$

As

$$
|\sigma(u)-\sigma(v)| \leq \frac{1}{u}(|u \sigma(u)-v \sigma(v)|+\delta|\sigma(v)|) \leq \frac{\delta}{u}+\frac{1}{u}|u \sigma(u)-v \sigma(v)|,
$$

it follows that

$$
|\sigma(u)-\sigma(v)| \leq \frac{\delta}{u} \log \frac{e u}{\delta}+\frac{1}{u} \int_{0}^{\infty} I(x) d x .
$$

By Cauchy's inequality

$$
\begin{aligned}
I(x)^{2} & \leq\left(4 \int_{0}^{u} e^{-2 t x} d t\right)\left(\int_{0}^{u}|t \widehat{\sigma}(t)-(t-\delta) \widehat{\sigma}(t-\delta)|^{2} e^{-2 t x} d t\right) \\
& \leq 2\left(\frac{1-e^{-2 x u}}{x}\right)\left(\int_{0}^{\infty}|t \widehat{\sigma}(t)-(t-\delta) \widehat{\sigma}(t-\delta)|^{2} e^{-2 t x} d t\right) .
\end{aligned}
$$

By Plancherel's formula the second term above is

$$
\begin{aligned}
& =\frac{1}{2 \pi} \int_{-\infty}^{\infty}|\mathcal{L}(t \widehat{\sigma}(t)-(t-\delta) \widehat{\sigma}(t-\delta), x+i y)|^{2} d y \\
& =\frac{1}{2 \pi} \int_{-\infty}^{\infty}|\mathcal{L}(t \widehat{\sigma}(t), x+i y)|^{2}\left|1-e^{-(x+i y) \delta}\right|^{2} d y
\end{aligned}
$$

From $(2.1)$ we see that $\mathcal{L}(t \widehat{\sigma}(t), x+i y)=\mathcal{L}(\widehat{\sigma}, x+i y) \mathcal{L}(\widehat{\chi}, x+i y)$ and so the above equals

$$
\begin{aligned}
& \frac{1}{2 \pi} \int_{-\infty}^{\infty}|\mathcal{L}(\widehat{\sigma}, x+i y) \mathcal{L}(\widehat{\chi}, x+i y)|^{2} \mid-\left.e^{-(x+i y) \delta}\right|^{2} d y \\
& \leq F(x)^{2} \cdot \frac{1}{2 \pi} \int_{-\infty}^{\infty}|\mathcal{L}(\widehat{\chi}, x+i y)|^{2} d y
\end{aligned}
$$

where

$$
F(x):=\max _{y \in \mathbb{R}}\left|1-e^{-(x+i y) \delta}\right||\mathcal{L}(\widehat{\sigma}, x+i y)|
$$


Now, by Plancherel's formula again,

$$
\frac{1}{2 \pi} \int_{-\infty}^{\infty}|\mathcal{L}(\widehat{\chi}, x+i y)|^{2} d y=\int_{0}^{\infty}|\widehat{\chi}(t)|^{2} e^{-2 t x} d t \leq \int_{0}^{u} e^{-2 t x} d t=\frac{1-e^{-2 x u}}{2 x}
$$

and so

$$
I(x) \leq \frac{1-e^{-2 x u}}{x} F(x) .
$$

We now demonstrate that $F(x)$ is a decreasing function of $x$. Suppose that $\beta>0$ is real, and recall that the Fourier transform of $k(z):=e^{-\beta|z|}$ is $\widehat{k}(\xi)=\int_{-\infty}^{\infty} e^{-\beta|z|-i \xi z} d z=2 \beta /\left(\beta^{2}+\xi^{2}\right)$. Hence

$$
e^{-\beta z}=k(z)=k(-z)=\frac{1}{\pi} \int_{-\infty}^{\infty} \frac{\beta}{\beta^{2}+\xi^{2}} e^{-i \xi z} d z
$$

by Fourier inversion for $z>0$. It follows that for $\delta+t>0$ we have

$$
\begin{aligned}
& \left(1-e^{-\delta(x+\beta+i y)}\right) e^{-t(x+\beta+i y)} \\
& \quad=\frac{1}{\pi} \int_{-\infty}^{\infty} \frac{\beta}{\beta^{2}+\xi^{2}} e^{-t(x+i y+i \xi)}\left(1-e^{-\delta(x+i y+i \xi)}\right) d \xi .
\end{aligned}
$$

Multiplying both sides by $\widehat{\sigma}(t)$, and integrating $t$ from 0 to $\infty$, we deduce that

$$
\begin{aligned}
\left(1-e^{-\delta(x+\beta+i y)}\right) & \mathcal{L}(\widehat{\sigma}, x+\beta+i y) \\
= & \frac{1}{\pi} \int_{-\infty}^{\infty} \frac{\beta}{\beta^{2}+\xi^{2}} \mathcal{L}(\widehat{\sigma}, x+i y+i \xi)\left(1-e^{-\delta(x+i y+i \xi)}\right) d \xi \\
\leq & \left(\max _{y \in \mathbb{R}}\left|\left(1-e^{-\delta(x+i y)}\right) \mathcal{L}(\widehat{\sigma}, x+i y)\right|\right) \frac{1}{\pi} \int_{-\infty}^{\infty} \frac{\beta}{\beta^{2}+\xi^{2}} d \xi
\end{aligned}
$$

and so $F(x+\beta) \leq F(x)$ as claimed. Therefore $F(x) \leq \lim _{x \rightarrow 0^{+}} F(x)$.

Now if $s=x+i y$ with $x>0$ then

$$
\begin{aligned}
\mathcal{L}\left(\frac{1-\chi(v)}{v}, s\right) & =\int_{0}^{\infty}\left(\frac{1-\chi(v) e^{-i v y}}{v}\right) e^{-v x} d v+\int_{0}^{\infty} \frac{e^{-v s}-e^{-v x}}{v} d v \\
& =\int_{0}^{\infty}\left(\frac{1-\chi(v) e^{-i v y}}{v}\right) e^{-v x} d v+\log (x / s),
\end{aligned}
$$

so that

$$
\mathcal{L}(\sigma, s)=\frac{1}{x} \exp \left(-\int_{0}^{\infty}\left(\frac{1-\chi(v) e^{-i v y}}{v}\right) e^{-v x} d v\right)
$$


Using this for $\widehat{\sigma}$ we have

$$
|\mathcal{L}(\widehat{\sigma}, x+i y)|=\frac{1}{x} \exp \left(-\int_{u}^{\infty} \frac{e^{-t x}}{t} d t-\int_{0}^{u} \operatorname{Re}\left(\frac{1-\chi(t) e^{-i t y}}{t}\right) e^{-t x} d t\right) .
$$

For $x \ll 1 / u$ we get

$$
\begin{aligned}
\int_{u}^{\infty} \frac{e^{-t x}}{t} d t & =\int_{u x}^{\infty} \frac{e^{-t}}{t} d t=\int_{1}^{\infty} \frac{e^{-t}}{t} d t+\int_{u x}^{1} \frac{e^{-t}-1}{t} d t+\log \frac{1}{u x} \\
& =-\gamma+\log \frac{1}{u x}+O(u x),
\end{aligned}
$$

since $\gamma=\int_{0}^{1} \frac{1-e^{-t}}{t} d t-\int_{1}^{\infty} \frac{e^{-t}}{t} d t$, so that

$$
|\mathcal{L}(\widehat{\sigma}, x+i y)|=e^{\gamma} u \exp \left(-\int_{0}^{u} \operatorname{Re}\left(\frac{1-\chi(t) e^{-i t y}}{t}\right) d t+O(u x)\right) .
$$

Note that this is $\ll_{u} 1$, so that the maximum of $\left|1-e^{-(x+i y) \delta}\right||\mathcal{L}(\widehat{\sigma}, x+i y)|$ cannot occur with $\|y \delta / 2 \pi\| \rightarrow 0$ as $x \rightarrow 0^{+}$(here $\|t\|$ denotes the distance from the nearest integer to $t$ ), else $F(x) \ll_{u} x+\|y \delta / 2 \pi\| \rightarrow 0$ as $x \rightarrow 0^{+}$, implying that $F(x)=0$, which is ridiculous. Thus the maximum occurs with $\|y \delta / 2 \pi\| \gg 1$ as $x \rightarrow 0^{+}$so that $1-e^{-(x+i y) \delta}=1-e^{-i y \delta}+O(x \delta)=$ $\left(1-e^{-i y \delta}\right)\{1+O(x \delta)\}$, so that

$$
\begin{aligned}
& \left|1-e^{-(x+i y) \delta}\right||\mathcal{L}(\widehat{\sigma}, x+i y)| \\
& =u\left|1-e^{-i y \delta}\right| \exp \left(\gamma-\int_{0}^{u} \operatorname{Re}\left(\frac{1-\chi(t) e^{-i t y}}{t}\right) d t+O(u x)\right) .
\end{aligned}
$$

Therefore $F(x) \leq u F\{1+O(u x)\}$ for sufficiently small $x$; and so $F(x) \leq u F$. Also $F(x) \leq 2 \max _{y \in \mathbb{R}}|\mathcal{L}(\widehat{\sigma}, x+i y)| \leq 2 / x$. Therefore, by (4.2), we get

$$
I(x) \leq \begin{cases}\frac{1-e^{-2 x u}}{x} u F & \text { if } x \leq 2 /(u F) \\ \frac{2}{x^{2}} & \text { if } x>2 /(u F)\end{cases}
$$

which when inserted in (4.1) yields the first estimate in the proposition.

Now if $F \leq 1$ then

$$
\int_{0}^{2 /(u F)} \frac{1-e^{-2 x u}}{x} d x \leq \int_{0}^{2 / u} \frac{1-e^{-2 x u}}{x} d x+\int_{2 / u}^{2 /(u F)} \frac{1}{x} d x \leq 2+\log (1 / F),
$$

and so we deduce the second estimate of Proposition 4.1. If $F>1$ this holds trivially since $|\sigma(u)-\sigma(v)| \leq 2$.

As an application of this proposition, we establish the following strangelooking Lipschitz estimate in the case that $\chi(t) \in[0,1]$ for all $t \geq 1$. 
Proposition 4.2. Let $\chi$ be a measurable function with $\chi(t)=1$ for $t \leq 1$ and $\chi(t) \in[0,1]$ for $t>1$, and let $\sigma$ denote the corresponding solution to (2.1). Let $1 \leq v \leq u$ be given and write $E(u)=(u /(u-v))^{\theta}$ for $\theta>0$. Then

$$
|\sigma(u)-\sigma(v)| \ll\left(\frac{u-v}{u}\right)^{\min \left\{1,1-\frac{1}{\pi} \sin (\pi \theta)\right\}}\left(1+\log \frac{u}{u-v}\right) .
$$

Proof. Let $\delta=u-v$ and $A=\int_{0}^{u} \frac{1-\chi(t)}{t} d t=\log E(u)$. We will show that

$$
\begin{aligned}
\exp \left(-\int_{0}^{u} \frac{1-\chi(t) \cos (t y)}{t} d t\right) \min (1, \delta y) & \\
& \ll\left(\frac{\delta}{u}\right)^{\min \left\{1,1-\frac{1}{\pi} \sin \left(\frac{\pi A}{\log (u / \delta)}\right)\right\}}
\end{aligned}
$$

for all positive $y$. The result then follows from Proposition 4.1 since $F \ll$ left side of (4.3).

If $y \leq e / u$ then the left side of (4.3) is $\leq e \delta / u$ and the result follows. Henceforth we may suppose that $y>e / u$. Since $\cos x=1+O\left(x^{2}\right)$, we get

$$
\int_{0}^{1 / y} \frac{1-\chi(t) \cos (t y)}{t} d t=\int_{0}^{1 / y} \frac{1-\chi(t)}{t} d t+O(1) .
$$

Thus if we let $z:=\int_{1 / y}^{u} \frac{1-\chi(t)}{t} d t$ then

$$
\begin{aligned}
\int_{0}^{u} \frac{1-\chi(t) \cos (t y)}{t} d t= & A-z+O(1)+\int_{1 / y}^{u} \frac{1-\chi(t) \cos (t y)}{t} d t \\
= & A-z+O(1)+\int_{1 / y}^{u} \frac{1-\cos (t y)}{t} d t \\
& +\int_{1 / y}^{u} \frac{1-\chi(t)}{t} \cos (t y) d t \\
= & A-z+\log (u y)+O(1)+\int_{1}^{u y} \frac{1-\chi(t / y)}{t} \cos t d t
\end{aligned}
$$

by making a change of variables, and since (integrating by parts)

$$
\int_{1 / y}^{u} \frac{\cos (t y)}{t} d t=\left.\frac{\sin (t y)}{y t}\right|_{1 / y} ^{u}+\int_{1 / y}^{u} \frac{\sin (t y)}{y t^{2}} d t=O(1) .
$$


By periodicity

$$
\int_{1}^{u y} \frac{1-\chi(t / y)}{t} \cos t d t=\int_{0}^{\pi} G(\theta) \cos \theta d \theta,
$$

where

$$
G(\theta):=\sum_{\substack{t \pm \theta \in 2 \pi \mathbb{Z} \\ 1 \leq t \leq u y}} \frac{1-\chi(t / y)}{t}
$$

and the sum over $t$ above is over real values of $t$ in the range [1,uy] such that $t \pm \theta$ is an integer multiple of $2 \pi$. Note that

$$
0 \leq G(\theta) \leq \frac{1}{\pi} \log (u y)+O(1) \quad \text { for all } \theta,
$$

and

$$
\int_{0}^{\pi} G(\theta) d \theta=\int_{1 / y}^{u} \frac{1-\chi(t)}{t} d t=z .
$$

Consider the problem of minimizing $\int_{0}^{\pi} G(\theta) \cos \theta d \theta$ over all functions $G$ satisfying these two constraints. Since $\cos \theta$ decreases from 1 to -1 in the range $[0, \pi]$, we see that this is achieved by taking $G(\theta)=0$ for $\theta \in\left[0, \pi-\theta_{0}\right]$, and $G(\theta)=\pi^{-1} \log (u y)+O(1)$ for $\theta \in\left[\pi-\theta_{0}, \pi\right]$, where $\theta_{0}$ satisfies $\theta_{0}\left(\pi^{-1} \log (u y)+O(1)\right)=z$. We conclude that

$$
\begin{aligned}
\int_{0}^{\pi} G(\theta) \cos \theta d \theta & \geq \int_{\pi-\theta_{0}}^{\pi} \cos \theta\left(\frac{1}{\pi} \log (u y)+O(1)\right) d \theta \\
& =-\frac{1}{\pi} \log (u y) \sin \theta_{0}+O(1) \\
& =-\frac{1}{\pi} \log (u y) \sin \left(\frac{\pi z}{\log (u y)+O(1)}\right)+O(1) \\
& =-\frac{1}{\pi} \log (u y) \sin \left(\frac{\pi z}{\log (u y)}\right)+O(1),
\end{aligned}
$$

since $0 \leq z \leq \log (u y)$. Therefore

$$
\begin{aligned}
& \int_{0}^{u} \frac{1-\chi(t) \cos (t y)}{t} d t \\
& \quad \geq A-z+\log (u y)\left(1-\frac{1}{\pi} \sin \left(\frac{\pi z}{\log (u y)}\right)\right)+O(1) .
\end{aligned}
$$

In the domain $0 \leq z \leq \log (u y)$, the right side of (4.4) is a non-increasing function of $z$, so that it is greater than the value with $z$ replaced by $\log (u y)$, that is, it is $>A+O(1)$. Therefore the left side of $(4.3)$ is $\ll e^{-A} \min (1, \delta y)$, which is $\leq \delta / u$ if $A \geq \log (u y)$, as required. If $A<\log (u y)$ then the 
right side of (4.4) is greater than the value with $z$ replaced by $A$, which is $\log (u y)-\frac{\log (u y)}{\pi} \sin (\pi A / \log (u y))+O(1)$, so that the left side of (4.3) is

$$
\ll \frac{\min (1, \delta y)}{u y}(u y)^{\frac{1}{\pi} \sin \left(\frac{\pi A}{\log (u y)}\right)} \text {. }
$$

This function is maximized when $y=1 / \delta$ in the range $\log (u y) \geq A$, at which point it yields the right side of (4.3), completing the proof.

Proof of Theorem 2. Let $\alpha=E(u)=e^{A}$. We may assume that $\alpha$ is large, and that $\sigma(u) \geq 1 / \alpha$, else our result follows trivially. Let $v=\left(1+e^{-\lambda}\right) u$ for some parameter $\lambda>A$, and select $\widehat{\chi}(t)=\chi(t)$ for $t \leq u$ and $\widehat{\chi}(t)=0$ for $t>u$, as earlier. Using Proposition 4.2 we deduce that there is a constant $C$ such that

$$
|\widehat{\sigma}(u)-\widehat{\sigma}(v)| \leq C(1+\lambda) \exp \left(-\lambda+\frac{\lambda}{\pi} \sin \left(\frac{\pi A}{\lambda}\right)\right) .
$$

If $\lambda \geq 2 A$, then this is $\leq C(1+\lambda) \exp (-\lambda(1-1 / \pi))$, which is easily verified to be $\leq 1 /(2 \alpha)$ if $\alpha$ is sufficiently large. If $A<\lambda \leq 2 A$, then the right side of $(4.5)$ is $\leq 2 C(1+A) \exp \left(-\lambda+\frac{\lambda}{\pi} \sin (\pi A / \lambda)\right)$, which is a decreasing function of $\lambda$ in our range. For $\lambda=A+\xi$ where $\xi:=c A^{2 / 3}(\log A)^{1 / 3}$, with $c>\left(6 / \pi^{2}\right)^{1 / 3}$, this equals

$$
\begin{aligned}
2 C(1+A) \exp (- & \left.A-\xi+\frac{A+\xi}{\pi} \sin \left(\frac{\pi A}{A+\xi}\right)\right) \\
= & 2 C(1+A) \exp \left(-A-\frac{\pi^{2}}{6} \frac{\xi^{3}}{A^{2}}+O\left(\frac{\xi^{4}}{A^{3}}\right)\right) \leq \frac{1}{2 \alpha}
\end{aligned}
$$

Thus we have proved that $|\widehat{\sigma}(u)-\widehat{\sigma}(v)| \leq 1 /(2 \alpha)$ for all $\lambda \geq A+\xi$, which implies that $\widehat{\sigma}(v) \geq 1 /(2 \alpha)$ for $u \leq v \leq u\left(1+e^{-A-\xi}\right)$. Therefore

$$
\frac{1}{u} \int_{u}^{\infty} \widehat{\sigma}(t) d t \geq \frac{1}{u} \int_{u}^{u\left(1+e^{-A-\xi}\right)} \widehat{\sigma}(v) d v \geq \frac{1}{u} \cdot u e^{-A-\xi} \cdot \frac{1}{2 \alpha}>\frac{1}{2 \alpha^{2} \exp (\xi)}
$$

which implies the theorem, by (3.2).

5. Determining $g(w)=\widetilde{g}(w)$ : preliminaries. In the remainder of the paper we will give an alternative, substantially shorter, proof of Hildebrand's result that $g(w)=\widetilde{g}(w)=\varrho(w)$. More precisely, we will establish the following theorem.

TheOREM 5. Let $\chi(t)=1$ for $t \leq 1$ and $\chi(t) \in[0,1]$ for all $t>1$, and let $\sigma(u)$ denote the corresponding solution to (2.1). Then $\sigma(u) \geq \varrho(E(u))$ for all $u$. Further if $1 \leq E(u) \leq 2$ and $\sigma(u)=\varrho(E(u))$ then $E(u / 2)=1$. If $E(u) \geq 2$ and $\sigma(u)=\varrho(E(u))$ then $E(u / E(u))=1$; that is, $\chi(t)=1$ for 
$t \leq u / E(u)$, and $\chi(t)=0$ for $u / E(u) \leq t \leq u$, except possibly on a set of measure 0 .

If $1 \leq E(u) \leq 2$ then using (2.5) with $n=0$ we see that $\sigma(u) \geq$ $1-I_{1}(u ; \chi)=1-\log E(u)=\varrho(E(u))$. Further (2.5) with $n=2$ gives

$$
\begin{aligned}
\sigma(u) & \geq 1-I_{1}(u ; \chi)+\frac{1}{2} I_{2}(u ; \chi)-\frac{1}{6} I_{3}(u ; \chi) \\
& \geq 1-\log E(u)+\frac{1}{2} I_{2}(u ; \chi)\left(1-\frac{\log E(u)}{3}\right)
\end{aligned}
$$

so that $\sigma(u)=\varrho(E(u))$ if and only if $I_{2}(u ; \chi)=0$, or in other words $E(u / 2)=1$. This proves Theorem 5 in the range $1 \leq E(u) \leq 2$ and we assume below that $E(u)>2$.

Henceforth we let $u_{0}:=u / E(u)<u_{1}:=u(1-1 / E(u))$. We also define

$$
B(u)=B_{\chi}(u)=\int_{0}^{u} \chi(v) d v .
$$

We note a simple principle that we shall use repeatedly.

Lemma 5.1. Let $b \geq a$ be real numbers. Let $f:[a, b] \rightarrow[0,1]$ and $g$ : $[a, b] \rightarrow \mathbb{R}$ be measurable functions, such that $g$ is non-decreasing in $[a, b]$, with $A:=\int_{a}^{b} f(t) d t$. Then

$$
\int_{a}^{a+A} g(t) d t \leq \int_{a}^{b} f(t) g(t) d t \leq \int_{b-A}^{b} g(t) d t .
$$

Proof. To prove the lower bound note that

$$
\begin{aligned}
\int_{a}^{b-A} f(t) g(t) d t & \leq g(b-A) \int_{a}^{b-A} f(t) d t=g(b-A) \int_{b-A}^{b}(1-f(t)) d t \\
& \leq \int_{b-A}^{b} g(t)(1-f(t)) d t
\end{aligned}
$$

and the result follows. The upper bound can be proved analogously.

Lemma 5.2. For all $0 \leq t \leq y$,

$$
y \frac{E(t)}{E(y)}-t \leq B(y)-B(t) \leq y-t \frac{E(y)}{E(t)} .
$$

Written differently,

$$
E(t) \leq \frac{E(y)}{y}(t+B(y)-B(t)) \quad \text { and } \quad E(y) \leq \frac{E(t)}{t}(y-B(y)+B(t)) .
$$


Proof. Note that

$$
\frac{E(y)}{E(t)}=\frac{y}{t} \exp \left(-\int_{t}^{y} \frac{\chi(v)}{v} d v\right) .
$$

Applying Lemma 5.1 (with $f(v)=\chi(v)$ and $g(v)=-1 / v$ ) we deduce that

$$
-\log \frac{t+B(y)-B(t)}{t} \leq-\int_{t}^{y} \frac{\chi(v)}{v} d v \leq-\log \frac{y}{y-B(y)+B(t)},
$$

and the lemma follows.

We note that

$$
B(y) \geq \frac{y}{E(y)}, \quad \frac{E(t)}{t} \geq \frac{E(y)}{y} \quad \text { for } 0 \leq t \leq y,
$$

which is a particular case of Lemma 5.2.

Our proof of Theorem 5 splits into two cases which we handle by different methods. The first case, which we treat in Section 6, is when either $E(u)$ is small $(\leq 2.6)$ or if $E\left(u_{0}\right) \geq E(u)-1$ is large. The other case concerns $E(u) \geq 2.6$ and $E\left(u_{0}\right)<E(u)-1$, which is handled in Section 7 .

6. The case $2<E(u) \leq 2.6$, or $E\left(u_{0}\right) \geq E(u)-1$

Proposition 6.1. If $E(u)>2$ and $E\left(u_{0}\right) \geq E(u)-1$ then $\sigma(u)>$ $\varrho(E(u))$.

Proof. Define $\widehat{\chi}(t)=\chi(t)$ for $t \leq u_{0}$ and $\widehat{\chi}(t)=1$ for $t>u_{0}$ and let $\widehat{\sigma}$ denote the solution to the corresponding integral equation. By Lemma 2.5 we have

$$
\begin{aligned}
\sigma(u)-\widehat{\sigma}(u) & =\sum_{j=1}^{\infty} \frac{(-1)^{j}}{j !} \int_{\substack{t_{1}, \ldots, t_{j} \geq u_{0} \\
t_{1}+\cdots+t_{j} \leq u}} \frac{1-\chi\left(t_{1}\right)}{t_{1}} \cdots \frac{1-\chi\left(t_{j}\right)}{t_{j}} \\
& \times \widehat{\sigma}\left(u-t_{1}-\cdots-t_{j}\right) d t_{1} \cdots d t_{j} \\
& \geq-\sum_{j \text { odd }} \frac{1}{j !}\left(\int_{u_{0}}^{u} \frac{1-\chi(t)}{t} d t\right)^{j}=-\frac{1}{2}\left(\frac{E(u)}{E\left(u_{0}\right)}-\frac{E\left(u_{0}\right)}{E(u)}\right) .
\end{aligned}
$$

Let $2 \leq n$ denote the largest even integer below $E(u)$. In the integral defining $I_{j}(u, \widehat{\chi})$ the integrand can be non-zero only if each $t_{i} \leq u_{0}$, so that $0 \leq I_{j}(u, \widehat{\chi}) \leq\left(\log E\left(u_{0}\right)\right)^{j}$ for all $j$. Also we have $t_{1}+\cdots+t_{j} \leq j u_{0} \leq$ $n u_{0} \leq u_{0} E(u)=u$ if $j \leq n$, implying that $I_{j}(u, \widehat{\chi})=\left(\log E\left(u_{0}\right)\right)^{j}$. Therefore by the inclusion-exclusion inequality $(2.5)$ we see that

$$
\widehat{\sigma}(u) \geq 1+\sum_{j=1}^{n+1} \frac{(-1)^{j}}{j !} I_{j}(u, \widehat{\chi}) \geq \sum_{j=0}^{n+1} \frac{(-1)^{j}}{j !}\left(\log E\left(u_{0}\right)\right)^{j} .
$$


Further note that

$$
\begin{aligned}
\frac{I_{n+2}(u, \widehat{\chi})}{(n+2) !}- & \frac{I_{n+3}(u, \hat{\chi})}{(n+3) !} \geq \frac{I_{n+2}(u, \hat{\chi})}{(n+2) !}\left(1-\frac{\log E\left(u_{0}\right)}{n+3}\right) \\
& \geq \frac{(\log E(u /(n+2)))^{n+2}}{(n+2) !}\left(1-\frac{\log E\left(u_{0}\right)}{n+3}\right) \\
& \geq \frac{\left(\log \left(E\left(u_{0}\right) E(u) /(n+2)\right)\right)^{n+2}}{(n+2) !}\left(1-\frac{\log E\left(u_{0}\right)}{n+3}\right),
\end{aligned}
$$

since $E(u /(n+2)) \geq E\left(u_{0}\right) u /\left((n+2) u_{0}\right)=E\left(u_{0}\right) E(u) /(n+2)$ by $(5.1)$. Thus another lower bound furnished by $(2.5)$ is

$$
\begin{aligned}
\widehat{\sigma}(u) \geq & \sum_{j=0}^{n+1} \frac{(-1)^{j}}{j !}\left(\log E\left(u_{0}\right)\right)^{j} \\
& +\frac{\left(\log \left(E\left(u_{0}\right) E(u) /(n+2)\right)\right)^{n+2}}{(n+2) !}\left(1-\frac{\log E\left(u_{0}\right)}{n+3}\right) .
\end{aligned}
$$

If $2 \leq E(u) \leq 6$ then using (6.1) together with (6.2b) for appropriate $n$ we check that $\sigma(u)>\varrho(E(u))$ if $E\left(u_{0}\right) \geq E(u)-1$. If $6 \leq n \leq E(u) \leq n+2$ then the right side of $(6.2 \mathrm{a})$ is at least $1 / E\left(u_{0}\right)-(\log (n+2))^{n+2} /(n+2)$ ! and combining this with (6.1) we get that for $E\left(u_{0}\right) \geq E(u)-1$,

$$
\begin{aligned}
\sigma(u) & \geq \frac{1}{2 E(u)(E(u)-1)}-\frac{1}{(n+2)(n+1)} \frac{(\log (n+2))^{n+2}}{n !} \\
& \geq \frac{.014}{(n+2)(n+1)}>\varrho(n) \geq \varrho(E(u)),
\end{aligned}
$$

since $(\log (n+2))^{n+2} / n ! \leq(\log 8)^{8} / 6 !<.486$ for $n \geq 6$ (note that $.14 / 56>$ $2 \cdot 10^{-4}$ whereas $\left.\varrho(6) \approx 2 \cdot 10^{-5}\right)$.

Henceforth we may assume that $E\left(u_{0}\right) \leq E(u)-1$. We complete this section by giving a proof of Theorem 5 for the range $2<E(u) \leq 2.6$.

Proposition 6.2. If $2<E(u) \leq 2.6$ then $\sigma(u) \geq \varrho(E(u))$ and equality holds only when $E\left(u_{0}\right)=1$.

Proof. By Proposition 6.1 we may assume that $e^{\xi}:=E(u / 3) \leq E\left(u_{0}\right) \leq$ $E(u)-1$ so that $\xi \leq \log (E(u)-1)$. Since one of $t_{1}, t_{2}$ or $t_{3}$ (in the definition of $\left.I_{3}\right)$ must be less than $u / 3$, we see easily that

$$
I_{3}(u) \leq 3\left(\int_{1}^{u / 3} \frac{1-\chi(v)}{v} d v\right) I_{2}(u) \leq 3 \xi I_{2}(u) .
$$

Thus using (2.5) with $n=2$ we get

$$
\sigma(u) \geq 1-I_{1}(u)+\frac{1}{2}(1-\xi) I_{2}(u)=1-\log E(u)+\frac{1}{2}(1-\xi) I_{2}(u) .
$$


Now $I_{1}(u-v) / v$ is a non-increasing function so by Lemma 5.1 we obtain

$$
\begin{aligned}
I_{2}(u) & \geq \int_{1}^{u / 3} \frac{1-\chi(v)}{v} I_{1}(u-v) d v+\int_{u / 3}^{u} \frac{1-\chi(v)}{v} I_{1}(u-v) d v \\
& \geq \int_{u /\left(3 e^{\xi}\right)}^{u / 3} I_{1}(u-v) \frac{d v}{v}+\int_{u e^{\xi} / E(u)}^{u} I_{1}(u-v) \frac{d v}{v} .
\end{aligned}
$$

Note that $I_{1}(t)=\log E(t) \geq \log E(u)-\int_{t}^{u} d v / v=\log (E(u) t / u)$, and also that $I_{1}(t) \geq \log E(u / 3)=\xi$ if $t \geq u / 3$. Using these bounds above we get

$$
\begin{aligned}
I_{2}(u) \geq & \int_{u /\left(3 e^{\xi}\right)}^{u / 3} \log \left(\frac{E(u)}{u}(u-v)\right) \frac{d v}{v} \\
& +\int_{u e^{\xi / E(u)}}^{u_{1}} \log \left(\frac{E(u)}{u}(u-v)\right) \frac{d v}{v}+\int_{u_{1}}^{2 u / 3} \xi \frac{d v}{v} .
\end{aligned}
$$

Let

$$
\gamma(E(u))=\int_{1}^{E(u)-1} \log (E(u)-t) \frac{d t}{t}=\int_{u_{0}}^{u_{1}} \log (E(u)-v E(u) / u) \frac{d v}{v} .
$$

We see that

$$
\begin{aligned}
I_{2}(u) \geq & \gamma(E(u))+\xi \log \left(\frac{2}{3} \frac{E(u)}{E(u)-1}\right) \\
& +\int_{u /\left(3 e^{\xi}\right)}^{u / 3} \log \left(\frac{E(u)}{u}(u-v)\right) \frac{d v}{v} \\
& -\int_{u / E(u)}^{u e^{\xi} / E(u)} \log \left(\frac{E(u)}{u}(u-v)\right) \frac{d v}{v} .
\end{aligned}
$$

After the changes of variables $v=u t /\left(3 e^{\xi}\right)$ and $v=u t / E(u)$, respectively, this becomes

$$
\begin{aligned}
& \gamma(E(u))+\xi \log \left(\frac{2}{3} \frac{E(u)}{E(u)-1}\right)+\int_{1}^{e^{\xi}} \log \left(\frac{E(u)\left(1-t /\left(3 e^{\xi}\right)\right)}{E(u)-t}\right) \frac{d t}{t} \\
& \geq \gamma(E(u))+\xi\left(\log \left(\frac{2}{3} \frac{E(u)}{E(u)-1}\right)+\log \left(\frac{E(u)\left(1-1 /\left(3 e^{\xi}\right)\right)}{E(u)-1}\right)\right),
\end{aligned}
$$

since $\log \left(\frac{E(u)\left(1-t /\left(3 e^{\xi}\right)\right)}{E(u)-t}\right)$ is an increasing function of $t$, as $3 e^{\xi}>3>E(u)$. 
Inserting the above bound for $I_{2}$ in (6.3) we deduce that

$$
\begin{aligned}
\sigma(u) \geq & \varrho(E(u))+\frac{\xi}{2}\left\{( 1 - \xi ) \left(\log \left(\frac{2}{3} \frac{E(u)}{E(u)-1}\right)\right.\right. \\
& \left.\left.+\log \left(\frac{E(u)\left(1-1 /\left(3 e^{\xi}\right)\right)}{E(u)-1}\right)\right)-\gamma(E(u))\right\},
\end{aligned}
$$

since $\varrho(x)=1-\log x+\gamma(x) / 2$ in the range $2 \leq x \leq 3$. Now, the quantity in \{\} is a decreasing function of $E(u)$ (since each term is), and so is bounded below by the value when substituting 2.6 in for $E(u)$, and this is positive for all $\xi \leq \log (1.6)$. It follows that $\sigma(u) \geq \varrho(E(u))$ and strict inequality holds unless $\xi=0$. If $\xi=0$ then $\sigma(u)=1-I_{1}(u)+I_{2}(u) / 2$ and if this equals $\varrho(E(u))$ then one must have $I_{2}(u)=\gamma(E(u))$, and arguing as above using Lemma 5.1, we see that this implies that $E\left(u_{0}\right)=1$.

7. The case $E\left(u_{0}\right) \leq E(u)-1$ and $E(u)>2.6$. We call $u$ a champion for $\sigma$ if the absolute minimum of $\sigma(v)-\varrho(E(v))$ in the interval $0 \leq v \leq u$ is attained at $u$. Evidently we need only establish Theorem 5 for champion $u$.

Proposition 7.1. If $u$ is a champion for $\sigma$ and

$$
u \varrho(E(u)) \leq \int_{0}^{u} \chi(t) \varrho(E(u-t)) d t
$$

then $\sigma(u) \geq \varrho(E(u))$. Further if strict inequality holds in (7.1) then $\sigma(u)>$ $\varrho(E(u))$.

Proof. Since $u$ is a champion for $\sigma$, we have

$$
\sigma(u)-\varrho(E(u)) \leq \sigma(v)-\varrho(E(v))
$$

for all $0 \leq v \leq u$. Multiplying both sides by $\chi(u-v)$ and then integrating with respect to $v$ from 0 to $u$, we obtain

$$
B(u)(\sigma(u)-\varrho(E(u))) \leq u \sigma(u)-\int_{0}^{u} \chi(v) \varrho(E(u-v)) d v \leq u(\sigma(u)-\varrho(E(u))),
$$

by $(2.1)$ and (7.1). The result follows as $B(u) \leq u$.

We will complete the proof of Theorem 5 by showing that (7.1) holds for $E(u)>2.6$ and $E\left(u_{0}\right) \leq E(u)-1$ and also determining when equality holds in (7.1). We define

and

$$
I_{1}=\int_{u_{1}}^{u} \varrho(E(u-t)) \chi(t) d t, \quad I_{2}=\int_{u_{0}}^{u_{1}} \varrho(E(u-t)) \chi(t) d t
$$

$$
I_{3}=\int_{0}^{u_{0}} \varrho(E(u-t)) \chi(t) d t
$$


Put $V=u-B\left(u_{0}\right)$. Note that $B\left(u_{0}\right) \leq u_{0}$ and so $u \geq V \geq u-u_{0}=u_{1}$.

Since $\varrho(E(u-t))$ is a non-decreasing function of $t$ we see by Lemma 5.1 that

$$
I_{3}=\int_{0}^{u_{0}} \chi(t) \varrho(E(u-t)) d t \geq \int_{0}^{B\left(u_{0}\right)} \varrho(E(u-t)) d t=\int_{V}^{u} \varrho(E(t)) d t .
$$

In the range $V \leq t \leq V E(u) / E(V)$ we have the bound $E(t) \leq E(V) t / V$, and in the range $V E(u) / E(V) \leq t \leq u$ we have the trivial bound $E(t) \leq E(u)$. Employing these bounds above we deduce that

$$
\begin{aligned}
I_{3} & \geq\left(u-\frac{V E(u)}{E(V)}\right) \varrho(E(u))+\int_{V}^{V E(u) / E(V)} \varrho\left(\frac{E(V)}{V} t\right) d t \\
& =\left(u-\frac{V E(u)}{E(V)}\right) \varrho(E(u))+\frac{V}{E(V)} \int_{E(V)}^{E(u)} \varrho(t) d t \\
& =u \varrho(E(u))-\frac{V}{E(V)} \int_{E(u)-1}^{E(V)} \varrho(t) d t .
\end{aligned}
$$

Next

$$
\begin{aligned}
I_{1} & \geq \varrho\left(E\left(u_{0}\right)\right)\left(B(u)-B\left(u_{1}\right)\right) \\
& \geq \frac{\varrho\left(E\left(u_{0}\right)\right)}{\varrho(E(u)-1)}\left(B(u)-B\left(u_{1}\right)\right) \varrho(E(u)-1) \\
& \geq \frac{V}{E(V)} \int_{E(u)-1}^{E\left(u_{1}\right)+\tau} \varrho(t) d t
\end{aligned}
$$

for $\tau$ satisfying

(7.3b) $\quad\left(E\left(u_{1}\right)+\tau\right)-(E(u)-1)=\frac{E(V)}{V} \frac{\varrho\left(E\left(u_{0}\right)\right)}{\varrho(E(u)-1)}\left(B(u)-B\left(u_{1}\right)\right)$.

By Lemma 5.2 and (5.1) we have

$$
\begin{aligned}
B(u)-B\left(u_{1}\right) & \geq \frac{u E\left(u_{1}\right)}{E(u)}-u_{1}=\frac{u}{E(u)}\left(E\left(u_{1}\right)-E(u)+1\right) \\
& \geq \frac{V}{E(V)}\left(E\left(u_{1}\right)-E(u)+1\right),
\end{aligned}
$$

and so $\tau \geq 0$ as $E\left(u_{0}\right) \leq E(u)-1$.

Finally note that

$$
I_{2} \geq \varrho\left(E\left(u_{1}\right)\right)\left(B\left(u_{1}\right)-B\left(u_{0}\right)\right) \geq \frac{V}{E(V)} \int_{E\left(u_{1}\right)+\tau}^{E\left(u_{1}\right)+\tau+\tau^{\prime}} \varrho(t) d t,
$$

where $\tau^{\prime}=(E(V) / V)\left(B\left(u_{1}\right)-B\left(u_{0}\right)\right)$. 
Combining the lower bounds given above for $I_{1}, I_{2}$ and $I_{3}$, we see that (7.1) follows provided $\tau+\tau^{\prime} \geq E(V)-E\left(u_{1}\right)$. Now let $C$ be a real number $\geq 1$ such that $\varrho\left(E\left(u_{0}\right)\right) \geq C \varrho(E(u)-1)$. Define $\eta:=u_{0} E(V) / V \geq 1$ by (5.1), and $\lambda:=E(u)-1 / E\left(u_{0}\right)$. By (5.1) we have $V=u-B\left(u_{0}\right) \leq u-u_{0} / E\left(u_{0}\right)=u_{0} \lambda$, and so $\eta \lambda \geq \eta V / u_{0}=E(V)$. Therefore if

$$
E\left(u_{0}\right) \geq \eta(1+C-C \eta) \lambda+1+\eta-E(u)
$$

then, by Lemma 5.2,

$$
\begin{aligned}
& \frac{V}{E(V)}\left(\tau+\tau^{\prime}+\left(E\left(u_{1}\right)-E(u)+1\right)\right) \\
& \quad=\frac{\varrho\left(E\left(u_{0}\right)\right)}{\varrho(E(u)-1)}\left(B(u)-B\left(u_{1}\right)\right)+\left(B\left(u_{1}\right)-B\left(u_{0}\right)\right) \\
& \quad \geq C\left(B(u)-B\left(u_{1}\right)\right)+\left(B\left(u_{1}\right)-B\left(u_{0}\right)\right) \\
& \quad \geq C(B(u)-B(V))+B(V)-B\left(u_{0}\right) \\
& \quad \geq C\left(u \frac{E(V)}{E(u)}-V\right)+V \frac{E\left(u_{0}\right)}{E(V)}-u_{0} \geq \frac{V}{E(V)}(E(V)-E(u)+1),
\end{aligned}
$$

so that $\tau+\tau^{\prime} \geq E(V)-E\left(u_{1}\right)$ as desired. Further if strict inequality holds in (7.5) then the inequality in (7.1) is also strict.

If $C \geq 1+1 / \lambda$ then the right side of (7.5) is decreasing in $\eta \geq 1$, so that it suffices to verify $(7.5)$ at $\eta=1$. This states that $E\left(u_{0}\right) \geq \lambda+2-$ $E(u)=2-1 / E\left(u_{0}\right)$, which always holds, further the inequality is strict unless $E\left(u_{0}\right)=1$. Consequently, if $\varrho\left(E\left(u_{0}\right)\right) / \varrho(E(u)-1) \geq E(u) /(E(u)-1)$ then criterion (7.1) follows, since $\lambda \geq E(u)-1$, and further (7.1) holds strictly unless $E\left(u_{0}\right)=1$.

If $C<1+1 / \lambda$, then the right side of (7.5) attains its maximum when $\eta=(C+1+1 / \lambda) /(2 C)$, so that (7.5) holds if

$$
E\left(u_{0}\right)+\frac{(C+1)^{2}}{4 C E\left(u_{0}\right)} \geq E(u) \frac{(C-1)^{2}}{4 C}+1+\frac{C+1}{2 C}+\frac{1}{4 C \lambda} .
$$

Taking $C=1$ and noting that $\lambda \geq 2.6-1 / E\left(u_{0}\right)$ we find that strict inequality in (7.6) holds (and thus strict inequality in (7.1)) if $E\left(u_{0}\right) \geq$ 1.4341. Hence we may assume that $E\left(u_{0}\right)<1.4341$. If $E(u) \geq 2.802$ then $\varrho\left(E\left(u_{0}\right)\right) / \varrho(E(u)-1) \geq \varrho(1.4341) / \varrho(1.802)>2.802 / 1.802 \geq E(u) /(E(u)-1)$ so that (7.1) holds (and equality there is possible only when $E\left(u_{0}\right)=1$ ). Hence we may assume that $2.6 \leq E(u)<2.802$.

Now take $C=\varrho(1.4341) / \varrho(1.6)>1$ so that (7.6) holds strictly (and thus (7.1) holds strictly) if $E\left(u_{0}\right) \geq 1.2383$. Hence we may assume that $E\left(u_{0}\right)<$ 1.2383. If $E(u) \geq 2.6635$ then $\varrho\left(E\left(u_{0}\right)\right) / \varrho(E(u)-1) \geq \varrho(1.2383) / \varrho(1.6635)$ $>2.6635 / 1.6635 \geq E(u) /(E(u)-1)$ so that $(7.1)$ holds (again with equality only when $\left.E\left(u_{0}\right)=1\right)$. Hence we may assume that $2.6 \leq E(u)<2.6635$. 
Now take $C=\varrho(1.2383) / \varrho(1.6)>1$ so that (7.6) holds strictly (and thus (7.1) strictly) if $E\left(u_{0}\right) \geq 1.0648$. Hence we may assume that $E\left(u_{0}\right)<1.0648$. If $E(u) \geq 2.6$ then $\varrho\left(E\left(u_{0}\right)\right) / \varrho(E(u)-1) \geq \varrho(1.0648) / \varrho(1.6)>2.6 / 1.6 \geq$ $E(u) /(E(u)-1)$ so that $(7.1)$ holds and with equality possible only when $E\left(u_{0}\right)=1$.

\section{References}

[1] P. Erdős and I. Ruzsa, On the small sieve. I. Sifting by primes, J. Number Theory 12 (1980), 385-394.

[2] A. Granville and K. Soundararajan, The spectrum of multiplicative functions, Ann. of Math. 153 (2001), 407-470.

[3] - - - Decay of mean values of multiplicative functions, Canad. J. Math. 55 (2003), 1191-1230.

[4] R. R. Hall, Halving an estimate obtained from Selberg's upper bound method, Acta Arith. 25 (1974), 347-351.

[5] A. Hildebrand, Quantitative mean value theorems for nonnegative multiplicative functions I, J. London Math. Soc. 30 (1984), 394-406.

[6] -, Quantitative mean value theorems for nonnegative multiplicative functions II, Acta Arith. 48 (1987), 209-260.

[7] —, Fonctions multiplicatives et équations intégrales, in: Séminaire de Théorie des Nombres de Paris, 1982-83, M.-J. Bertin (ed.), Birkhäuser, 1984, 115-124.

[8] E. Wirsing, Das asymptotische Verhalten von Summen über multiplikative Funktionen II, Acta Math. Acad. Sci. Hungar. 18 (1967), 411-467.

Département de mathématiques et de statistique

Université de Montréal

CP 6128 succ. Centre-Ville

Montréal, QC H3C 3J7, Canada

E-mail: andrew@dms.umontreal.ca
Department of Mathematics University of Michigan Ann Arbor, MI 48109, U.S.A.

E-mail: ksound@umich.edu 